\title{
Efficacy and Safety of Direct-acting Antivirals in Hepatitis C Virus-infected Patients Taking Proton Pump Inhibitors
}

\author{
Karn Wijarnpreecha*1, Supavit Chesdachai ${ }^{2}$, Charat Thongprayoon ${ }^{1}$, \\ Veeravich Jaruvongvanich ${ }^{3}$, Patompong Ungprasert ${ }^{4}$ and Wisit Cheungpasitporn ${ }^{4}$
}

\begin{abstract}
${ }^{1}$ Department of Internal Medicine, Bassett Medical Center, Cooperstown, NY, USA; ${ }^{2}$ Department of Medicine, Faculty of Medicine Ramathibodi Hospital, Mahidol University, Bangkok, Thailand; ${ }^{3}$ Department of Internal Medicine, University of Hawaii, Honolulu, HI, USA; ${ }^{4}$ Department of Internal Medicine, Mayo Clinic, Rochester, MN, USA
\end{abstract}

\begin{abstract}
Background and Aims: Direct-acting antiviral (DAA) therapy is the cornerstone of the treatment of chronic hepatitis $C$ virus (HCV) infection. Eradication of $\mathrm{HCV}$, predicted by the attainment of a sustained virologic response (SVR) 12 weeks following DAA therapy, is the goal of this treatment. Interestingly, recent studies have reported the possible association between HCV-infected patients with DAA therapy concomitant use of proton pump inhibitors (PPIs) and lower odds of achieving SVR. This meta-analysis was conducted to summarize all available data and to estimate this potential association. Methods: Comprehensive literature review was conducted by first searching the Medline and Embase databases through March 2017 to identify all studies that investigated the safety and efficacy of DAAs in patients with HCV infection taking PPIs versus those without PPIs. Adjusted point estimates from each study were combined by the generic inverse variance method of DerSimonian and Laird. Results: Nine cohort studies with 32,684 participants met the eligibility criteria and were included in the meta-analysis. The use of PPIs concomitant with DAAs among HCV-infected patients was associated with lower odds of achieving SVR compared with non-PPI users (pooled odds ratio (OR): 0.74 , $95 \%$ confidence interval $(\mathrm{CI}): 0.63-0.88, \mathrm{I}^{2}=24 \%$ ). Subgroup analysis addressed the association between PPIs use and SVR12 demonstrated the association of PPI users showing lower odds of achieving SVR12 compared with those with no use of PPIs (pooled OR: $0.68,95 \% \mathrm{CI}$ : $0.51-0.9, \mathrm{I}^{2}=$ $33 \%)$. Conclusions: This study demonstrated a significantly increased risk of failure to achieve SVR in HCV-infected patients taking DAA with PPIs compared to non-PPI users. Providers should consider whether PPI therapy is indicated for patients and withdraw of PPI therapy in the absence of indications, especially while on DAA therapy.
\end{abstract}

Keywords: Hepatitis C; Antiviral agents; Proton pump inhibitors; Sustained virologic response; Meta-analysis.

Abbreviations: BMI, body mass index; $\mathrm{CI}$, confidence interval; DAA, directacting antiviral; DSV, dasabuvir; EBR, elbasvir; GZR, grazopravir; $\mathrm{HCV}$, hepatitis $\mathrm{C}$ virus; HR, hazard ratio; IL28B, interleukin-28B gene; $\mathrm{kPa}$, kilopascal; LDV, ledipasvir; mg, milligram; OBV, ombitasvir; OR, odds ratio; PPI, proton pump inhibitor; $\mathrm{PTV} / \mathrm{r}$, paritaprevir/ritonavir; $\mathrm{RBV}$, ribavirin; $\mathrm{RNA}$, ribonucleic acid; $\mathrm{RR}$, relative risk; SIR, standardized incidence ratio; SOF, sofosbuvir; SVR, sustained virologic response.

Received: 6 March 2017; Revised: 24 June 2017; Accepted: 12 July 2017

*Correspondence to: Karn Wijarnpreecha, Department of Internal Medicine, Bassett Medical Center, One Atwell Road, Cooperstown, NY 13326, USA. Tel: +1-607-547-4805, Fax: +1-604-547-6612, E-mail: dr.karn.wi@gmail.com
Citation of this article: Wijarnpreecha $\mathrm{K}$, Chesdachai $\mathrm{S}$, Thongprayoon C, Jaruvongvanich V, Ungprasert P, Cheungpasitporn W. Efficacy and safety of direct-acting antivirals in hepatitis $C$ virus-infected patients taking proton pump inhibitors. J Clin Transl Hepatol 2017;5(4):327-334. doi: 10. 14218/JCTH.2017.00017.

\section{Introduction}

Hepatitis $\mathrm{C}$ virus ( $\mathrm{HCV}$ ) infection is one of the leading causes of cirrhosis and estimated to affect more than 185 million people worldwide. ${ }^{1}$ Direct-acting antiviral (DAA) therapy is the cornerstone of the treatment of chronic HCV infection. The vast majority of $\mathrm{HCV}$-infected patients can be cured with current DAA treatment. ${ }^{2-5}$ It has been shown that eradication of HCV is associated with such benefits as decreased overall mortality, improved quality of life, and reduced healthcare utilization. ${ }^{6,7}$ Therefore, the goal of the treatment is to eradicate HCV RNA, predictable by attainment of a sustained virologic response (SVR; defined as undetectable of RNA level 12 weeks following the completion of DAA therapy).

Proton pump inhibitors (PPIs) are one of the most commonly prescribed medications worldwide for the treatment of all acid-related disorders, including gastroesophageal reflux disease and peptic ulcer. All DAA therapy can interact with PPIs, which affect gastric pH and can affect DAA bioavailability, thereby leading to sub-therapeutic levels of antiviral drugs and possibly to failure to achieve SVR. ${ }^{8-11}$ In fact, recent epidemiologic studies have reported the possible association between HCV-infected patients with DAA therapy concomitant use PPIs and lower odds of achieving SVR compared to non-PPI users. ${ }^{8,10-17}$ However, the results are still inconsistent. Thus, this systematic review and meta-analysis was conducted to summarize all available evidence with the aim of better characterizing this relationship.

\section{Methods}

\section{Information sources and search strategy}

A systematic literature search was conducted using the Embase and Medline databases from inception to March 2017 to identify all original studies that investigated the safety and efficacy of DAAs in patients with HCV infection taking PPIs versus those without PPIs. The systematic 
literature review was independently conducted by three investigators (K.W., S.C., and W.C.) using the search strategy that included the terms for "hepatitis C", "direct-acting antivirals", "sustained virologic response", and "proton pump inhibitors" as described in Online Supplementary Data 1. A manual search for additional potentially relevant studies was also performed using references of the included articles. No language limitation was applied. This study was conducted in agreement with the Preferred Reporting Items for Systematic Reviews and Meta-Analysis (commonly known as PRISMA) statement which is provided as Online Supplementary Data 2.

\section{Selection criteria}

Eligible studies were required to be case-control, cross-sectional or cohort studies that had investigated the safety and efficacy of DAAs in patients with HCV infection taking PPIs. They must provide the effect estimates (odds ratios (ORs), relative risks (RRs), hazard ratios (HRs) or standardized incidence ratio (SIR)) with $95 \%$ confidence intervals (CIs). Inclusion was not restricted by study size. When more than one article using the same database/cohort was available, the study with the most comprehensive data/analyses was included.

Retrieved articles were independently reviewed for their eligibility by the same three investigators. Any discrepancy was resolved by conference with all investigators. NewcastleOttawa quality assessment scale was used to appraise the quality of study in three areas, including the recruitment of cases and controls, the comparability between the two groups and the ascertainment of the outcome of interest for cohort study and the exposure for case-control study. ${ }^{18}$

\section{Data abstraction}

A structured data collection form was used to extract the following data from each study: title of the study, publication year, name of the first author, year of the study, country where the study was conducted, number of participants, demographic data of participants, definition of PPIs use, type of DAA therapy, outcome measurement, adjusted effect estimates with $95 \%$ CIs and covariates that were adjusted in the multivariable analysis. To ensure accuracy, this data extraction process was independently performed by two investigators ( $\mathrm{KW}$ and $\mathrm{WC}$ ) and was reviewed by the senior investigator (WC).

\section{Statistical analysis}

Data analysis was performed using the Comprehensive MetaAnalysis Software (version 2.2.064; Biostat Inc). Adjusted point estimates from each study were combined by the generic inverse variance method of DerSimonian and Laird, which assigned the weight of each study in the pooled analysis based on its variance. ${ }^{19}$ In light of the high likelihood of between-study variance due to the different study populations, DAA therapy and definition of PPI use, therefore a random-effect model was used. Cochran's $Q$ test and $\mathrm{I}^{2}$ statistic were used to determine the between-study heterogeneity. A value of $\mathrm{I}^{2}$ of $0-25 \%$ represented insignificant heterogeneity, $26-50 \%$ represented low heterogeneity, $51-75 \%$ represented moderate heterogeneity, and more than $75 \%$ represented high heterogeneity. ${ }^{20}$ Meta-regression was performed to assess the effect of DAA regimens (sofosbuvir (SOF) based vs. non-SOF-based regimens) and the use of ribavirin (RBV) on the SVR using a random-effects metaregression. ${ }^{21}$ Egger's regression symmetry test was used to assess for publication bias. A $p$-value less than 0.05 was considered statistically significant for all analysis.

\section{Results}

Seven hundred and twenty potentially eligible articles were identified using our search strategy (251 articles from Medline and 469 articles from Embase). After the exclusion of 251 duplicate articles, 469 articles underwent title and abstract review. Four hundred and fifty-six articles were excluded at this stage since they were case reports, correspondences, review articles or interventional studies, leaving 13 articles for full-text review. Two of those were excluded after the full-length review as they did not report the outcome of interest, while one article was excluded since for being a descriptive study without comparative analysis. Ten studies met our eligibility criteria. However, two studies used the same database. ${ }^{11,22}$ To avoid duplication, we excluded one of those studies, and we decided to exclude the study that had been published as an abstract ${ }^{22}$ and later published as an original article (which was more comprehensive and had a larger number of participants). ${ }^{11}$ Therefore, nine cohort studies with 32,684 participants met the eligibility criteria. $8,10-17$ The literature retrieval, review, and selection process are shown in Fig. 1. The main features and quality assessment of the studies included in this meta-analysis are shown in Table 1.

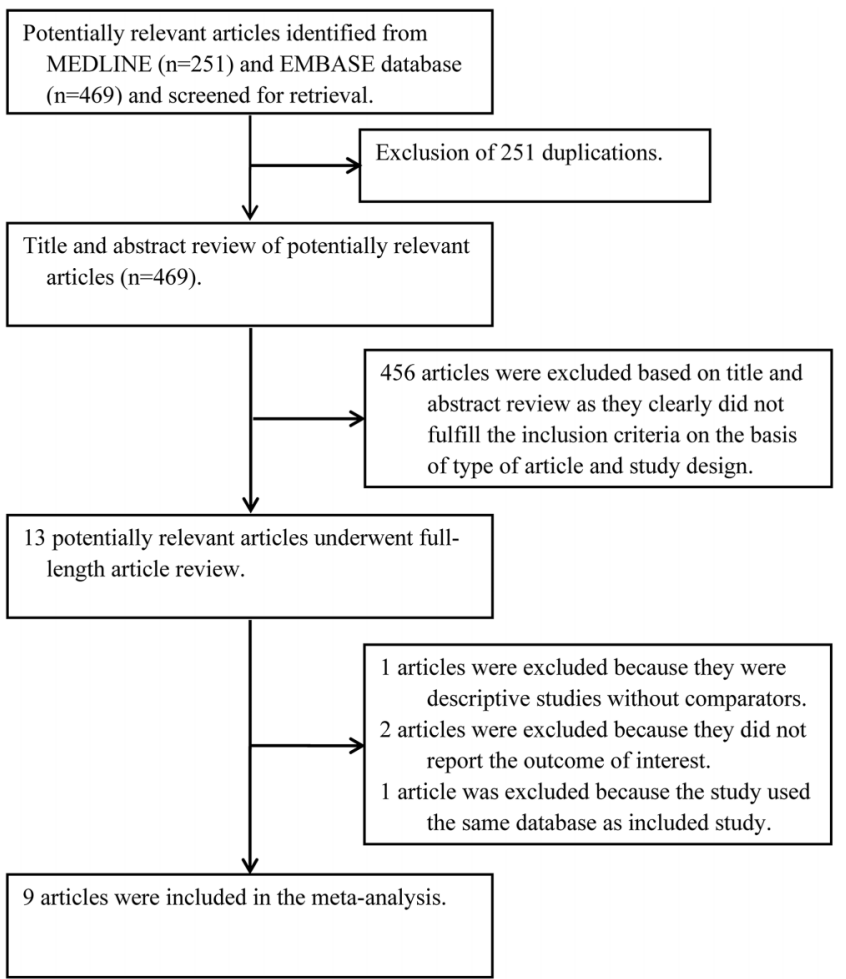

Fig. 1. Literature review process. 


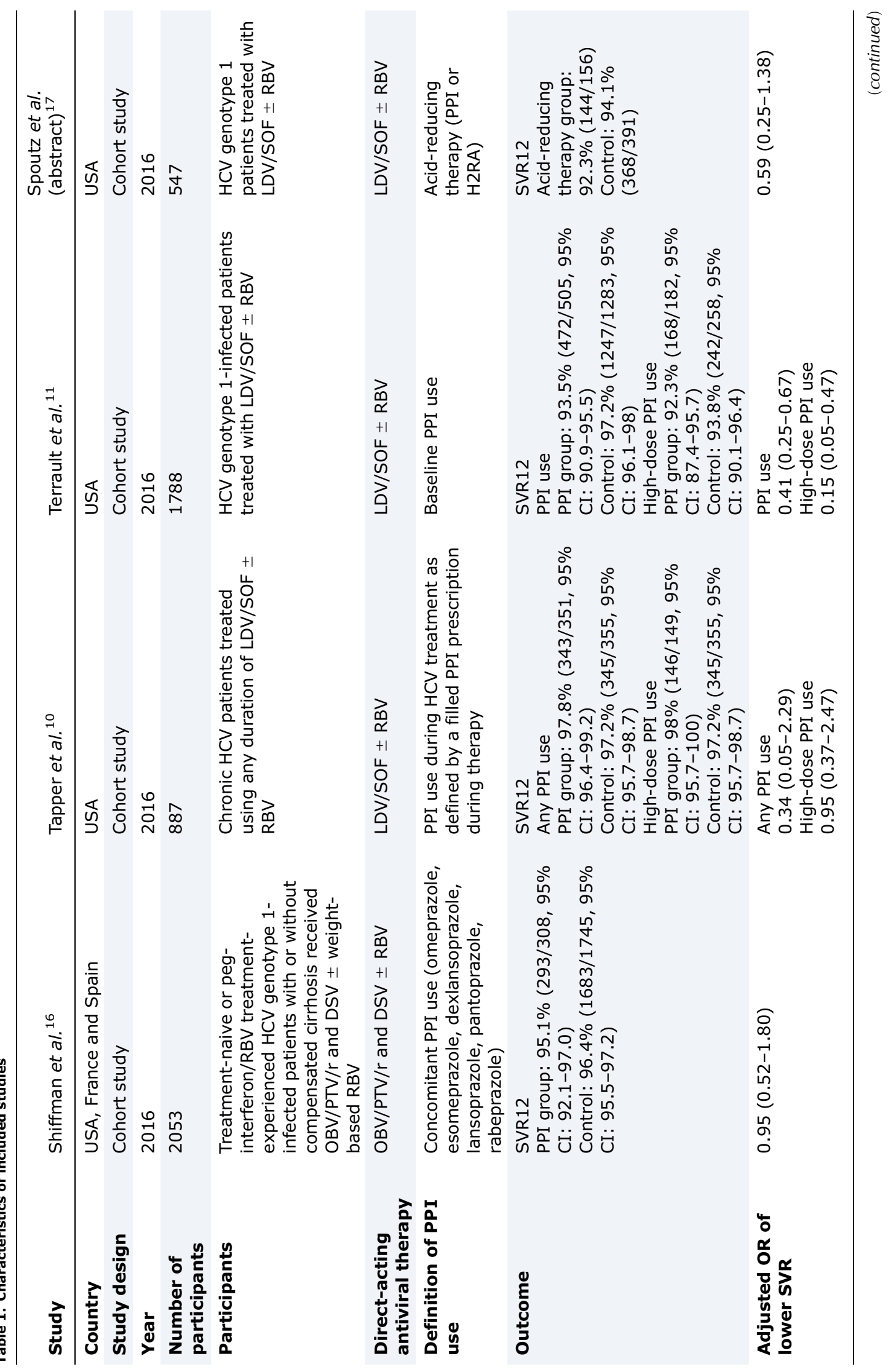









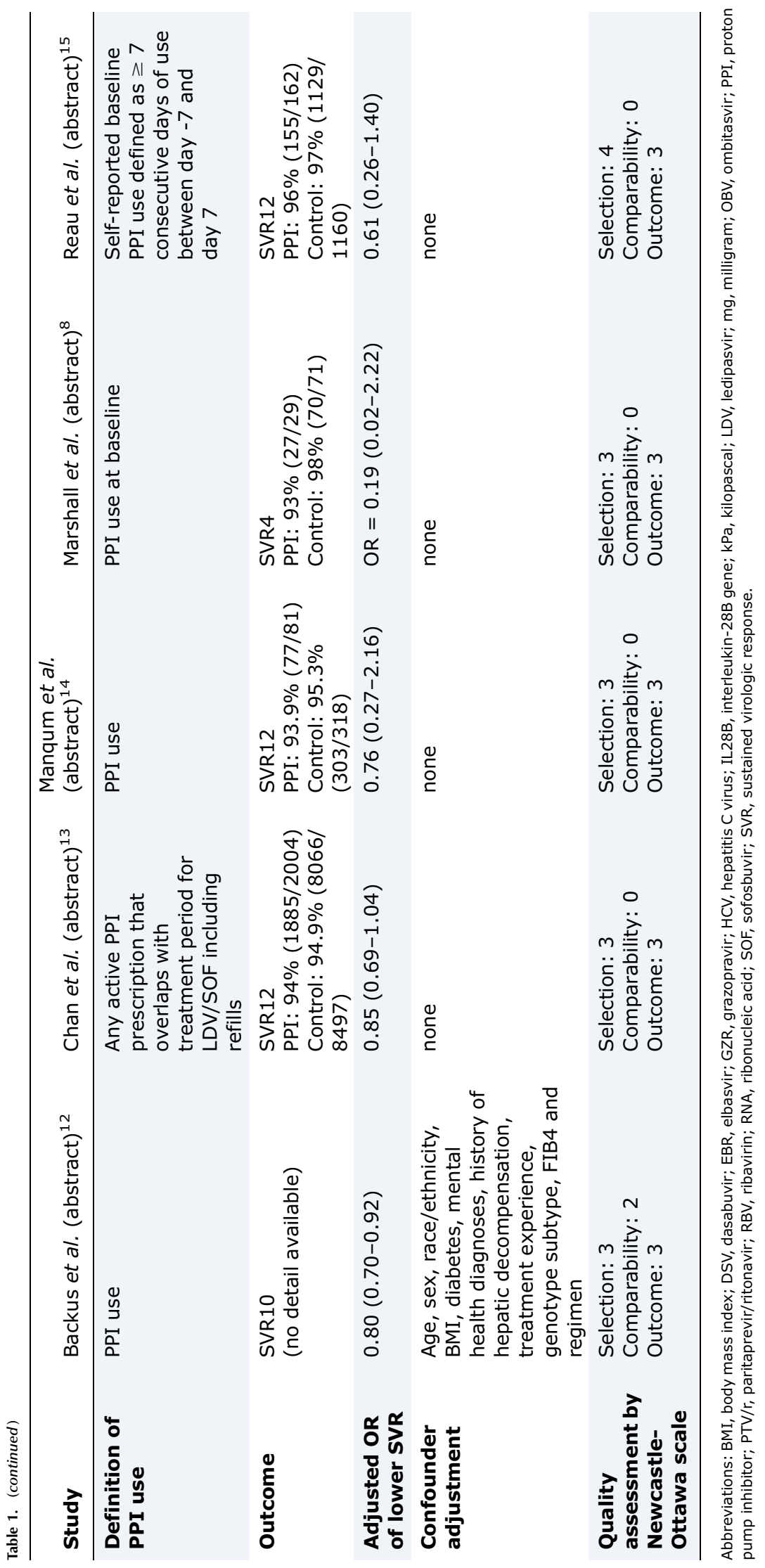


We found that the use of PPIs concomitant with DAAs among HCV-infected patients was associated with lower odds of achieving SVR compared with non-PPI users (pooled OR of $0.74,95 \%$ CI: $0.63-0.88, p<0.001$ ), as shown in Fig. 2 . The heterogeneity between studies of the overall analysis was insignificant, with an $\mathrm{I}^{2}$ of $24 \%$. Subgroup analysis to address the association between PPI uses and SVR12 also demonstrated the association of PPI users with lower odds of achieving SVR12 compared with those with no use of PPI (pooled OR of $0.68,95 \% \mathrm{CI}: 0.51-0.9, p=0.01$ ), as shown in Fig. 3. The heterogeneity between studies of the overall analysis was low, with an $\mathrm{I}^{2}$ of $33 \%$. Moreover, subgroup analysis restricted to only the studies with adjusted confounding factors also showed an association between PPI uses with lower odds of achieving SVR compared with non-PPI users (pooled OR of $0.66,95 \% \mathrm{CI}: 0.47-0.94, p=0.02$ ), as shown in Fig. 4. The heterogeneity between studies of the overall analysis was low, with an $\mathrm{I}^{2}$ of $50 \%$.

\section{Meta-regression analysis}

Meta-regression showed no significant impact of the uses of SOF $(p=0.16)$ or RBV $(p=0.18)$ in DAA regimens on the association between PPI use and lower odds of achieving SVR12.

\section{Evaluation for publication bias}

There was no publication bias for the overall included studies, as assessed by funnel plotting (Fig. 5) and the Egger's regression asymmetry test ( $p=0.24$ ) of the association between PPI use and lower odds of achieving SVR.

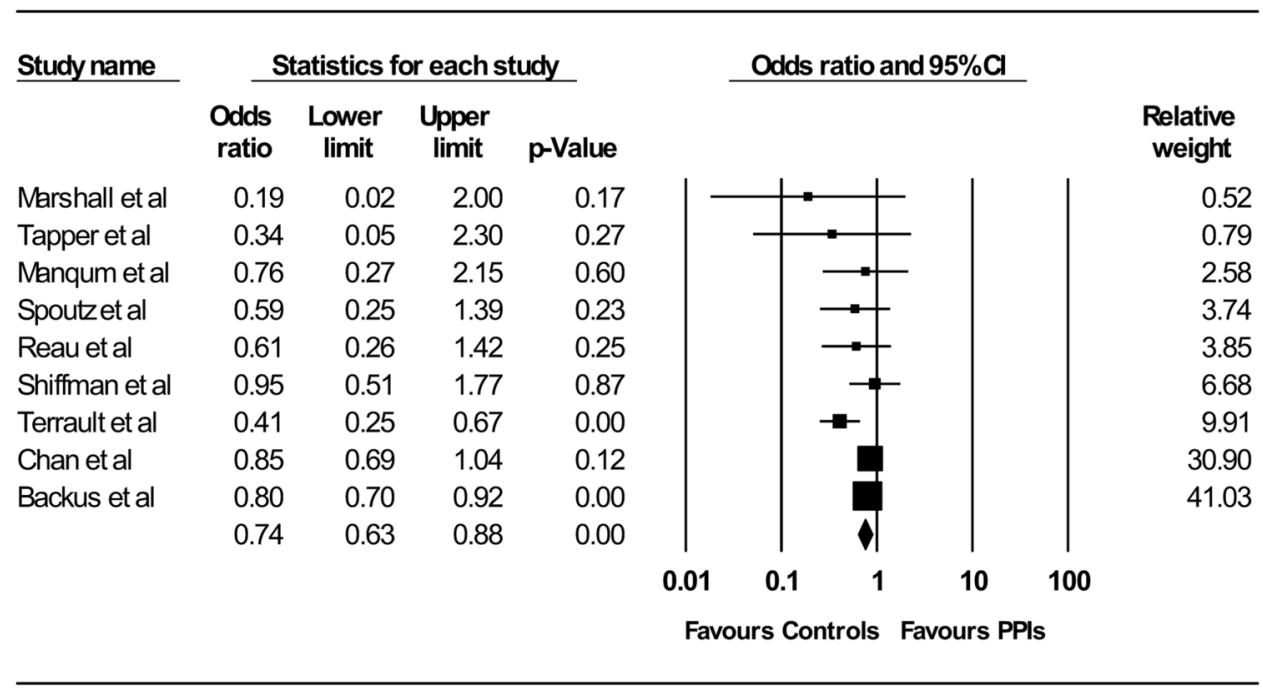

Meta Analysis

Fig. 2. Forest plot of the overall included studies.

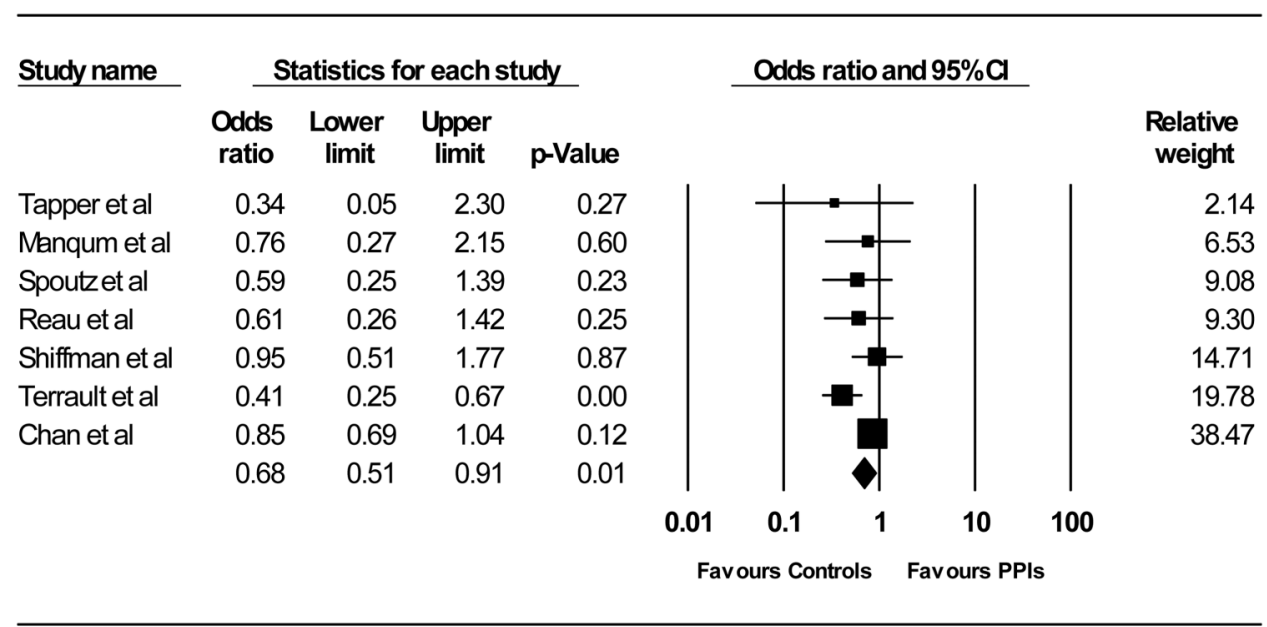

Meta Analysis

Fig. 3. Forest plot of the included studies reported the outcome of SVR12. 
Wijarnpreecha K. et al: PPIs and DAA in HCV

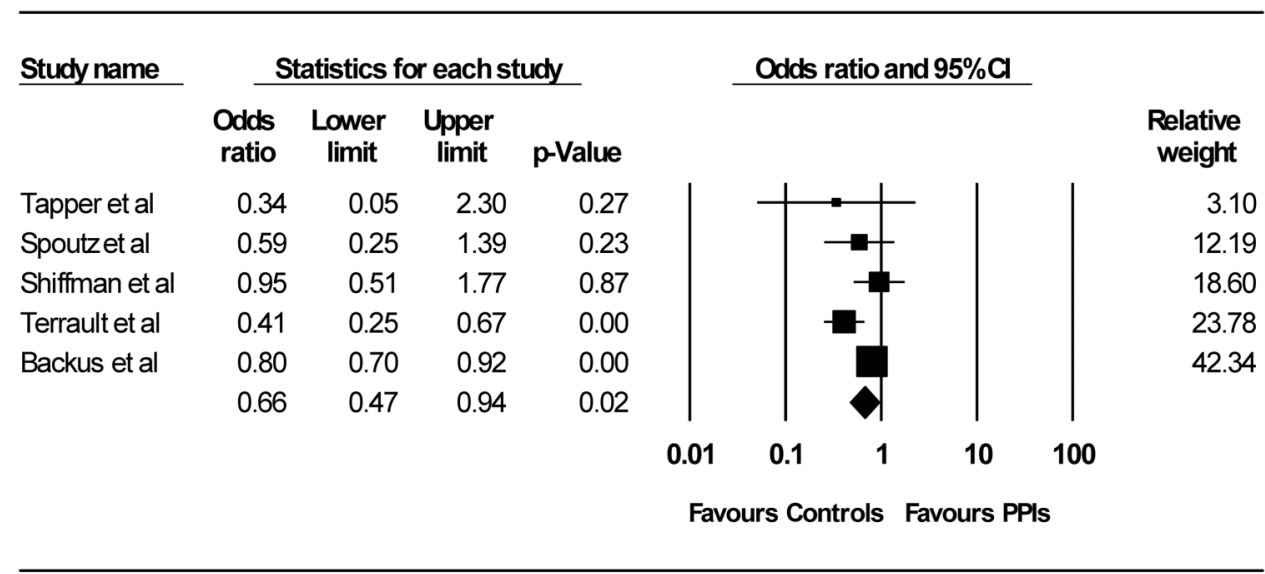

Meta Analysis

Fig. 4. Forest plot of the included studies with confounder adjustment.

\section{Discussion}

To the best of our knowledge, this is the first systematic review and meta-analysis that summarized all available studies that have reported on the efficacy and safety of DAA therapy in HCV-infected patients taking PPIs. DAA therapies in combination with PPIs may result in increasing risk of failure to achieve SVR. We found an approximately 1.4 -fold increased risk of failure to achieve SVR12 within the PPI users group compared to the non-PPI users group.

The true pathogenesis of this association is still unclear; however, there is potential explanation. Ledipasvir's solubility decreases as gastric $\mathrm{pH}$ increases. Thus, acid reducing agents can affect drug absorption and drug level. ${ }^{23,24}$ The package label for ledipasvir (LDV) recommended that patients who take PPIs should not take a dose higher than omeprazole $20 \mathrm{mg}$ daily or equivalent and taken fasting at the same time as LDV/SOF. Whether the patients can follow these recommendations is unknown and may affect the result of the real world data and this meta-analysis. Seven of nine studies in this meta-analysis reported the interaction of PPIs and the LDV/SOF regimen. ${ }^{8,10-14,17}$ Only one study by Terrault et al. ${ }^{11}$ showed a significantly decreased achievement of SVR among PPI users daily. Tapper et al. ${ }^{10}$ showed that twice daily PPI use

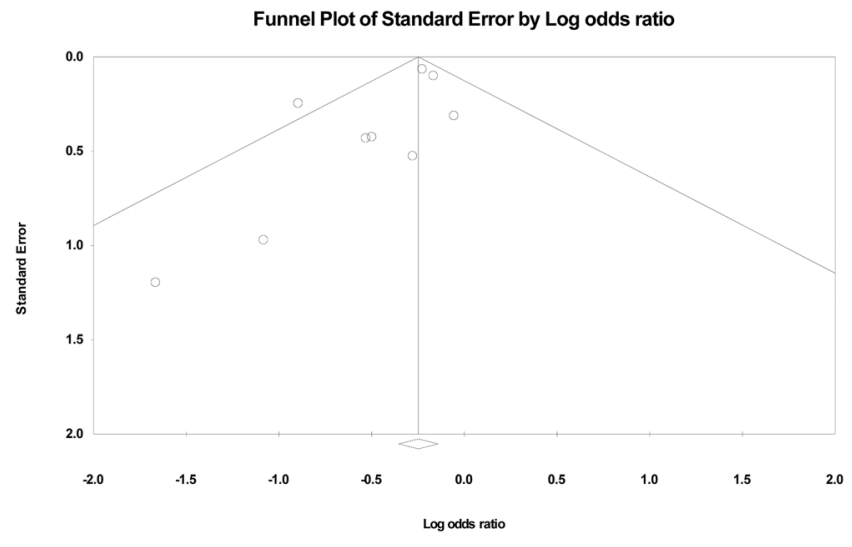

Fig. 5. Funnel plot of the overall included studies. was associated with lower odds ratio for SVR but not daily PPI use. This can imply that PPI use decreased odds of achieving SVR, especially twice daily PPI usage.

The systematic literature review process of this study was comprehensive, and the quality of included studies was good even though some of the included studies are abstracts. Moreover, the statistical heterogeneity of this meta-analysis was low. We acknowledge, however, that this study had some limitations and, thus, the results should be interpreted with caution. First, not all included studies used pharmacy records to confirm which patients filled their PPI prescriptions throughout the treatment course, as well as the dose, quantity of the pills dispensed, and the frequency. The method to define and measure PPI use by pharmacy records is better than that for data from PPI use at baseline. Thus, we may not know the data on the dose, frequency and refilled prescription of PPIs throughout the course of the treatment in most of the included studies. Besides, most of the included studies were abstracts and not yet published in full original studies. Therefore, the final data and report of each study may change, such as the number of participants and adjusted confounding factors analysis. However, we believe that the primary outcome of each study which focused on the efficacy and safety of PPI use among HCV-infected patients with DAA therapy will not change from the report of the published abstracts.

In summary, this study demonstrated a significantly increased risk of failure of achievement of SVR in HCVinfected patients taking DAA with PPIs compared to non-PPI users. Providers should consider whether PPI therapy is indicated for these patients and withdraw PPI therapy in the absence of indications.

\section{Conflict of interest}

The authors have no conflict of interests related to this publication.

\section{Author contributions}

Contributed to conception and design of the study, and critical revision (KW, WC), acquisition of data, analysis and 
interpretation of data ( $\mathrm{KW}, \mathrm{SC}, \mathrm{CT}, \mathrm{VJ})$, manuscript writing $(\mathrm{KW}, \mathrm{SC})$, revised the article ( $\mathrm{CT}, \mathrm{VJ})$. All authors approved the final revision.

\section{References}

[1] Mohd Hanafiah K, Groeger J, Flaxman AD, Wiersma ST. Global epidemiology of hepatitis $C$ virus infection: new estimates of age-specific antibody to HCV seroprevalence. Hepatology 2013;57:1333-1342. doi: 10.1002/hep.26141.

[2] Afdhal N, Reddy KR, Nelson DR, Lawitz E, Gordon SC, Schiff E, et al. Ledipasvir and sofosbuvir for previously treated HCV genotype 1 infection. N Engl J Med 2014;370:1483-1493. doi: 10.1056/NEJMoa1316366.

[3] Afdhal N, Zeuzem S, Kwo P, Chojkier M, Gitlin N, Puoti M, et al. Ledipasvir and sofosbuvir for untreated HCV genotype 1 infection. N Engl J Med 2014;370: 1889-1898. doi: 10.1056/NEJMoa1402454.

[4] Feld JJ, Jacobson IM, Hézode C, Asselah T, Ruane PJ, Gruener N, et al. Sofosbuvir and Velpatasvir for HCV Genotype 1, 2, 4, 5, and 6 Infection. N Engl J Med 2015;373:2599-2607. doi: 10.1056/NEJMoa1512610.

[5] Foster GR, Afdhal N, Roberts SK, Bräu N, Gane EJ, Pianko S, et al. Sofosbuvir and Velpatasvir for HCV Genotype 2 and 3 Infection. N Engl J Med 2015;373: 2608-2617. doi: 10.1056/NEJMoa1512612.

[6] Younossi ZM, Singer ME, Mir HM, Henry L, Hunt S. Impact of interferon free regimens on clinical and cost outcomes for chronic hepatitis $C$ genotype 1 patients. J Hepatol 2014;60:530-537. doi: 10.1016/j.jhep.2013.11.009.

[7] Younossi ZM, Stepanova M, Marcellin P, Afdhal N, Kowdley KV, Zeuzem S, et al. Treatment with ledipasvir and sofosbuvir improves patient-reported outcomes: Results from the ION-1, -2 , and -3 clinical trials. Hepatology 2015;61:1798-1808. doi: 10.1002/hep.27724.

[8] Marshall V, Hirsch AA, Rife K, Silveira M, Compan A, Moreland AL, et al. Impact of proton pump inhibitors on achieving SVR from 8 week course of ledipasvir/sofosbuvir. Gastroenterology 2016;150:S1095. doi: 10.1016/ S0016-5085(16)33698-8.

[9] Sahloff EG, Duggan JM. Clinical outcomes associated with concomitant use of atazanavir and proton pump inhibitors. Ann Pharmacother 2006;40: 1731-1736. doi: 10.1345/aph.1H217.

[10] Tapper EB, Bacon BR, Curry MP, Dieterich DT, Flamm SL, Guest LE, et al. Evaluation of proton pump inhibitor use on treatment outcomes with ledipasvir and sofosbuvir in a real-world cohort study. Hepatology 2016;64: 1893-1899. doi: 10.1002/hep.28782.

[11] Terrault NA, Zeuzem S, Di Bisceglie AM, Lim JK, Pockros PJ, Frazier LM, et al. Effectiveness of ledipasvir-sofosbuvir combination in patients with hepatitis $C$ virus infection and factors associated with sustained virologic response. Gastroenterology 2016;151:1131-1140.e5. doi: 10.1053/j.gastro.2016. 08.004 .
[12] Backus L, Belperio P, Shahoumian T, Loomis TP, Mole L. Predictors of sustained virologic response in 21,242 genotype 1 patients treated with ledipasvir/ sofosbuvir \pm ribavirin or ombitasvir/paritaprevir/ritonavir+dasabuvir \pm ribavirin in routine medical practice. Hepatology 2016;64:1000A.

[13] Chan AW, Bryan WE, Hashem MA, Townsend ML, Wang LA, Choi SS, et al. Hepatitis $\mathrm{C}(\mathrm{HCV})$ virologic outcomes in veterans taking ledipasvir/sofosbuvir with concomitant acid suppressing medication. Hepatology 2016;64:468A.

[14] Mangum JE, Pillai AA, Wedd JP, Todd SB. Sustained virologic response (SVR) in patients taking ledipasvir-sofosbuvir (LDV/SOF) and a proton pump inhibitor (PPI) in the treatment of chronic hepatitis $\mathrm{C}$ virus (HCV). Hepatology 2016;64:1004A.

[15] Reau N, Robertson M, Feng HP, Caro L, Yeh WW, Nguyen BYT, et al. Concomitant proton pump inhibitor use does not reduce the efficacy of elbasvir/grazoprevir. Hepatology 2016;64:429A-430A.

[16] Shiffman ML, Rustgi V, Bennett M, Forns X, Asselah T, Planas Vila R, et al. Safety and efficacy of ombitasvir/paritaprevir/ritonavir plus dasabuvir with or without ribavirin in $\mathrm{HCV}$-infected patients taking concomitant acid-reducing agents. Am J Gastroenterol 2016;111:845-851. doi: 10.1038/ajg.2016.108.

[17] Spoutz P, Schaefer M, Pandya PK, Patterson M. The impact of concomitant acid reducing therapy (ART) on sustained virologic response (SVR-12) among genotype 1 infected chronic hepatitis $\mathrm{C}(\mathrm{CHC})$ patients treated with ledipasvir/sofosbuvir (LDV/SOF) with or without ribavirin. Hepatology 2016; 64:477A-478A.

[18] Stang A. Critical evaluation of the Newcastle-Ottawa scale for the assessment of the quality of nonrandomized studies in meta-analyses. Eur J Epidemiol 2010;25:603-605. doi: 10.1007/s10654-010-9491-z.

[19] DerSimonian R, Laird N. Meta-analysis in clinical trials. Control Clin Trials 1986;7:177-188. doi: 10.1016/0197-2456(86)90046-2.

[20] Higgins JP, Thompson SG, Deeks J], Altman DG. Measuring inconsistency in meta-analyses. BMJ 2003;327:557-560. doi: 10.1136/bmj.327.7414.557.

[21] Kelley GA, Kelley KS. Statistical models for meta-analysis: A brief tutorial. World J Methodol 2012:2:27-32. doi: 10.5662/wjm.v2.i4.27.

[22] Terrault N, Zeuzem S, Di Bisceglie AM, Lim JK, Pockros PJ, Frazier LM, et al. Treatment Outcomes With 8, 12 and 24 Week Regi- mens of Ledipasvir/ Sofosbuvir for the Treatment of Hep- atitis C Infection: Analysis of a Multicenter Prospective, Observational Study. Hepatology 2015;62:256A.

[23] Cuenca-Lopez F, Rivero A, Rivero-Juárez A. Pharmacokinetics and pharmacodynamics of sofosbuvir and ledipasvir for the treatment of hepatitis C. Expert Opin Drug Metab Toxicol 2017;13:105-112. doi: 10.1080/17425255. 2017.1255725.

[24] Dick TB, Lindberg LS, Ramirez DD, Charlton MR. A clinician's guide to drugdrug interactions with direct-acting antiviral agents for the treatment of hepatitis C viral infection. Hepatology 2016;63:634-643. doi: 10.1002/ hep. 27920 . 